\title{
A NON-EXTENDABLE BOUNDED LINEAR MAP BETWEEN $C^{*}$-ALGEBRAS
}

\author{
NARUTAKA OZAWA \\ Texas A\&M University, College Station, TX77843, USA and \\ University of Tokyo, Komaba, 153-8914, Japan (ozawa@math.tamu.edu)
}

(Received 10 September 1999)

\begin{abstract}
We present an example of a $C^{*}$-subalgebra $A$ of $\mathbb{B}(H)$ and a bounded linear map from $A$ to $\mathbb{B}(K)$ which does not admit any bounded linear extension. This generalizes the result of Robertson and gives the answer to a problem raised by Pisier. Using the same idea, we compute the exactness constants of some Q-spaces. This solves a problem raised by Oikhberg. We also construct a Q-space which is not locally reflexive.
\end{abstract}

Keywords: bounded linear extensions; non-exact Q-spaces

AMS 2000 Mathematics subject classification: Primary 46L05

Secondary 46L07

\section{A non-extendable bounded linear map}

Definition 1.1. Let $X$ be a closed subspace of a Banach space $Y$. We say $X$ is complemented in $Y$ if there is a bounded linear projection from $Y$ onto $X$. We say $X$ is weakly complemented in $Y$ if there is a bounded linear map $T$ from $Y$ to $X^{* *}$ such that $\left.T\right|_{X}=\iota_{X}$, where $\iota_{X}$ is the canonical inclusion map of $X$ into $X^{* *}$.

Let $A$ be a $C^{*}$-algebra. If there is a faithful $*$-representation $A \subset \mathbb{B}(H)$ such that $A$ is (weakly) complemented in $\mathbb{B}(H)$, then it follows from the injectivity of $\mathbb{B}(H)$ that for any faithful *-representation $A \subset \mathbb{B}(K), A$ is (weakly) complemented in $\mathbb{B}(K)$. A $C^{*}$-algebra $A$ has the weak expectation property (WEP) $[\mathbf{1 3}]$ if for every faithful $*$-representation $A \subset \mathbb{B}(H)$, there is a complete contraction $T: \mathbb{B}(H) \rightarrow A^{* *}$ such that $\left.T\right|_{A}=\iota_{A}$.

Lemma 1.2. Let $A$ be a $C^{*}$-algebra and let $A \subset \mathbb{B}(H)$ be the universal representation, i.e. $\bar{A}^{\text {ultraweak }}=A^{* *}$. If $A$ is weakly complemented in $\mathbb{B}(H)$ and is locally reflexive, then $A^{* *}$ is complemented in $\mathbb{B}(H)$.

Proof. Let $T: \mathbb{B}(H) \rightarrow A^{* *}$ be a bounded linear map such that $\left.T\right|_{A}=\operatorname{id}_{A}$. Let $I$ be a set of all pairs $i=(E, F)$ consisting of finite-dimensional subspaces $E$ of $A^{* *}$ and $F$ of $\mathbb{B}(H)_{*} . I$ is then directedly ordered by inclusions. Fix $i=(E, F)$ in $I$. Since $A$ is locally reflexive, there is a map $S_{i}: E \rightarrow A$ with $\left\|S_{i}\right\|_{\mathrm{cb}} \leqslant 1+(1 / \operatorname{dim}(E))$ such that 
$\left\langle S_{i}(e), f\right\rangle=\langle e, f\rangle$ for all $e \in E$ and $f \in F$. Since $\mathbb{B}(H)$ is injective, we can extend $S_{i}$ to $\bar{S}_{i}: \mathbb{B}(H) \rightarrow \mathbb{B}(H)$ with $\left\|\bar{S}_{i}\right\|_{\mathrm{cb}} \leqslant\left\|S_{i}\right\|_{\mathrm{cb}}$. Define $T_{i}: \mathbb{B}(H) \rightarrow A^{* *}$ by $T_{i}=T \circ \bar{S}_{i}$. We then have $\lim \sup \left\|T_{i}\right\| \leqslant\|T\|$ and $\lim \left\langle T_{i}(e), f\right\rangle=\langle e, f\rangle$ for all $e \in A^{* *}$ and $f \in \mathbb{B}(H)_{*}$. Let $\tilde{T}: \mathbb{B}(H) \rightarrow A^{* *}$ be a cluster point of the net $\left\{T_{i}\right\}_{i}$ in the point-ultraweak topology. $\tilde{T}$ is then the desired bounded linear projection.

Next we use results due to De Cannière and Haagerup and due to Kirchberg. Let $C_{\lambda}^{*}\left(\mathbb{F}_{n}\right)$ be the reduced group $C^{*}$-algebra of the free group $\mathbb{F}_{n}$ with $n$ generators $(n \geqslant 2)$. Then, by $[\mathbf{3}], C_{\lambda}^{*}\left(\mathbb{F}_{n}\right)$ has the complete metric approximation property. Thus, $C_{\lambda}^{*}\left(\mathbb{F}_{n}\right)$ is exact and a fortiori is locally reflexive [12]. By [11], there are a $C^{*}$-algebra $B$ with the WEP and a surjective $*$-homomorphism $\pi$ from $B$ onto $C_{\lambda}^{*}\left(\mathbb{F}_{n}\right)$. Since $C_{\lambda}^{*}\left(\mathbb{F}_{n}\right)$ has the metric approximation property, there is a contractive linear lifting $\varphi: C_{\lambda}^{*}\left(\mathbb{F}_{n}\right) \rightarrow B$, i.e. $\pi \circ \varphi=\operatorname{id}_{C_{\lambda}^{*}\left(\mathbb{F}_{n}\right)}$. (There is even a unital $k$-positive lifting for each $k \in \mathbb{N}$ (see $[20])$.) We need one more ingredient due to Haagerup and Pisier. Let $\operatorname{VN}\left(\mathbb{F}_{n}\right)$ be the group von Neumann algebra of the free group $\mathbb{F}_{n}$ with $n$ generators $(n \geqslant 2)$ and let $V N\left(\mathbb{F}_{n}\right) \subset \mathbb{B}(K)$ be any faithful $*$-representation. $\mathrm{VN}\left(\mathbb{F}_{n}\right)$ is then not complemented in $\mathbb{B}(K)$ (see Corollary 4.9 in $[\mathbf{8}]$ ).

Lemma 1.3. Let $C_{\lambda}^{*}\left(\mathbb{F}_{n}\right) \subset \mathbb{B}(H)$ be any faithful $*$-representation. Then $C_{\lambda}^{*}\left(\mathbb{F}_{n}\right)$ is not weakly complemented in $\mathbb{B}(H)$.

Proof. If $C_{\lambda}^{*}\left(\mathbb{F}_{n}\right)$ is weakly complemented in $\mathbb{B}(H)$, then by Lemma 1.2 and the preceding remarks, $C_{\lambda}^{*}\left(\mathbb{F}_{n}\right)^{* *}$ is complemented in $\mathbb{B}(K)$ for any faithful $*$-representation $C_{\lambda}^{*}\left(\mathbb{F}_{n}\right)^{* *} \subset \mathbb{B}(K)$. Since the group von Neumann algebra $\operatorname{VN}\left(\mathbb{F}_{n}\right)$ is complemented in $C_{\lambda}^{*}\left(\mathbb{F}_{n}\right)^{* *}$, a fortiori it is complemented in $\mathbb{B}(K)$. This contradicts Corollary 4.9 in [8].

Theorem 1.4. Let $C_{\lambda}^{*}\left(\mathbb{F}_{n}\right) \subset \mathbb{B}(H)$ be a faithful *-representation. Let $B \subset \mathbb{B}(K)$ be a $C^{*}$-subalgebra with the WEP and $\pi$ be a surjective $*$-homomorphism from $B$ onto $C_{\lambda}^{*}\left(\mathbb{F}_{n}\right)$. If $\varphi: C_{\lambda}^{*}\left(\mathbb{F}_{n}\right) \rightarrow B$ is a bounded linear lifting of $\pi$, then there is no bounded linear extension $\bar{\varphi}: \mathbb{B}(H) \rightarrow \mathbb{B}(K)$ of $\varphi$.

Proof. Suppose that there is a bounded linear extension $\bar{\varphi}: \mathbb{B}(H) \rightarrow \mathbb{B}(K)$ of $\varphi$. Since $B$ has the WEP, there is a bounded linear map $\psi: \mathbb{B}(K) \rightarrow B^{* *}$ such that $\left.\psi\right|_{B}=\iota_{B}$. Let us define $T: \mathbb{B}(H) \rightarrow C_{\lambda}^{*}\left(\mathbb{F}_{n}\right)^{* *}$ by $T=\pi^{* *} \circ \psi \circ \bar{\varphi}$. $T$ is then a bounded linear map and $\left.T\right|_{C_{\lambda}^{*}\left(\mathbb{F}_{n}\right)}=\iota_{C_{\lambda}^{*}\left(\mathbb{F}_{n}\right)}$. This contradicts Lemma 1.3.

Remark 1.5. Since $C_{\lambda}^{*}\left(\mathbb{F}_{n}\right)$ is weakly complemented in $\mathrm{VN}\left(\mathbb{F}_{n}\right)$ (see the proof of Lemma 7.6 in $[\mathbf{1 1}])$, there is a bounded linear map from $\operatorname{VN}\left(\mathbb{F}_{n}\right)$ to $\mathbb{B}(K)$ without bounded linear extension to $\mathbb{B}\left(\ell_{2}\left(\mathbb{F}_{n}\right)\right)$.

Problem 1.6. Let $A$ be a $C^{*}$-subalgebra of $\mathbb{B}(H)$ and assume that any bounded linear map from $A$ to $\mathbb{B}(H)$ extends to a bounded linear map on $\mathbb{B}(H)$. Is $A$ weakly complemented in $\mathbb{B}(H)$ ? 


\section{Computing exactness constants of some Q-spaces}

We will compute the exactness constant of some Q-spaces. A Q-space is a quotient operator space of a minimal operator space. By the duality between maximal and minimal operator spaces, a dual operator space of a subspace of a maximal operator space is a Q-space. See $[\mathbf{1}]$ and $[\mathbf{2}]$ for details.

Let $E$ be a finite-dimensional operator space. For any $C^{*}$-algebra $B$ and any closed twosided ideal $J$ in $B$, there is a canonical isomorphism $T_{E}:(E \otimes B) /(E \otimes J) \rightarrow E \otimes(B / J)$, where $\otimes$ means the minimal tensor product. Let $C$ be a constant. We say $E$ is $C$ exact if $\left\|T_{E}^{-1}\right\| \leqslant C$ for all choices of $B$ and $J$. By the canonical isometric identification $E \otimes X=C B\left(E^{*}, X\right)$ for an operator space $X$ (see $\left.[\mathbf{2}, \mathbf{4}]\right), E$ is $C$-exact if any complete contraction from the dual operator space $E^{*}$ to any quotient $C^{*}$-algebra $B / J$ has a lifting with cb-norm $\leqslant C$. For an infinite-dimensional operator space $X$, we say $X$ is $C$-exact if every finite-dimensional operator subspace of $X$ is $C$-exact and we say $X$ is exact if it is $C$-exact for some constant $C$. The exactness constant $\operatorname{ex}(X)$ of $X$ is defined by $\operatorname{ex}(X)=\inf \{C: X$ is $C$-exact $\}$. See [17] for details.

Define an operator space $E_{n} \subset \mathbb{M}_{n} \oplus \mathbb{M}_{n} \subset \mathbb{M}_{2 n}$ by $E_{n}=\operatorname{span}\left\{e_{k 1} \oplus e_{1 k}: \quad k=\right.$ $1,2, \ldots, n\}$, where $\left\{e_{j k}\right\}$ is a standard matrix unit in $\mathbb{M}_{n}$. By Proposition 1.3 in [8], there are two maps $w: E_{n} \rightarrow C_{\lambda}^{*}\left(\mathbb{F}_{n}\right)$ and $v: C_{\lambda}^{*}\left(\mathbb{F}_{n}\right) \rightarrow E_{n}$ such that $v \circ w=\operatorname{id}_{E_{n}}$ and $\|v\|_{\mathrm{cb}} \leqslant 2,\|w\|_{\mathrm{cb}} \leqslant 1$. By Lemma 4.2 in [8], any projection $P$ from $\mathbb{M}_{2 n}$ onto $E_{n}$ has cb-norm $\geqslant \frac{1}{2}(1+\sqrt{n})$. By Smith's lemma (Theorem 2.1 in $[\mathbf{2 1}]$ ), we have $\|P\|_{\mathrm{cb}}=\|P\|_{2 n}$ for any map $P: \mathbb{M}_{2 n} \rightarrow E_{n}$. Hence, by a standard averaging argument (see $[\mathbf{8}, \mathbf{2 0}]$ ), we have $\|P\| \geqslant \frac{1}{2}(1+\sqrt{n})$ for any projection $P$ from $\mathbb{M}_{2 n}\left(\mathbb{M}_{2 n}\right)$ onto $\mathbb{M}_{2 n}\left(E_{n}\right)$. On the other hand, by Remark 4.3 in [8], there is a projection $Q$ from $\mathbb{M}_{2 n}\left(\mathbb{M}_{2 n}\right)$ onto $\mathbb{M}_{2 n}\left(E_{n}\right)$ with $\|Q\|=\frac{1}{2}(1+\sqrt{n})$. See $[8]$ for details.

Theorem 2.1. We equip $\mathbb{M}_{2 n}\left(E_{n}\right)$ with a new operator space structure induced by the canonical embedding into $\max \left(\mathbb{M}_{2 n}\left(\mathbb{M}_{2 n}\right)\right)$ and denote the resultant operator space by $F_{n}$, i.e. $F_{n}=\mathbb{M}_{2 n}\left(E_{n}\right)$ as a Banach space and $F_{n} \subset \max \left(\mathbb{M}_{2 n}\left(\mathbb{M}_{2 n}\right)\right)$ as an operator space. $F_{n}^{*}$ is then a $4 n^{3}$-dimensional $Q$-space such that

$$
\frac{1}{4}(1+\sqrt{n}) \leqslant \operatorname{ex}\left(F_{n}^{*}\right) \leqslant \| \text { id: } \min \left(F_{n}^{*}\right) \rightarrow F_{n}^{*} \|_{\mathrm{cb}} \leqslant \frac{1}{2}(1+\sqrt{n}) .
$$

Proof. We note that the formal identity $J: F_{n} \rightarrow \mathbb{M}_{2 n}\left(E_{n}\right)$ is completely contractive. Let $v$ and $w$ be as in the preceding remarks and let $\tilde{w}: F_{n} \rightarrow \mathbb{M}_{2 n}\left(C_{\lambda}^{*}\left(\mathbb{F}_{n}\right)\right)$ be a complete contraction defined by $\tilde{w}=\left(\operatorname{id}_{\mathbb{M}_{2 n}} \otimes w\right) \circ J$. By [11], there are a $C^{*}$-algebra $B$ with the WEP and a surjective $*$-homomorphism $\pi$ from $B$ onto $\mathbb{M}_{2 n}\left(C_{\lambda}^{*}\left(\mathbb{F}_{n}\right)\right)$. Suppose that $F_{n}^{*}$ is $C$-exact. By definition, there is a lifting $\varphi: F_{n} \rightarrow B$ of $\tilde{w}$ with $\|\varphi\|_{\text {cb }} \leqslant C$. Since $B$ has the WEP, $\varphi$ extends to $\bar{\varphi}: \max \left(\mathbb{M}_{2 n}\left(\mathbb{M}_{2 n}\right)\right) \rightarrow B^{* *}$ with $\|\bar{\varphi}\|_{\mathrm{cb}} \leqslant C$. Let us define $P: \mathbb{M}_{2 n}\left(\mathbb{M}_{2 n}\right) \rightarrow \mathbb{M}_{2 n}\left(E_{n}\right)$ by $P=\left(\operatorname{id}_{\mathbb{M}_{2 n}} \otimes v\right)^{* *} \circ \pi^{* *} \circ \bar{\varphi}$. $P$ is then a projection with $\|P\| \leqslant 2 C$. Thus, we have $C \geqslant \frac{1}{4}(1+\sqrt{n})$. This proves the first inequality. Since $\operatorname{ex}\left(\min \left(F_{n}^{*}\right)\right)=1$, we have the second inequality.

Next, let $Q$ be a projection as in the preceding remarks. We then have

$\|$ id: $F_{n} \rightarrow \max \left(F_{n}\right)\left\|_{\mathrm{cb}} \leqslant\right\| Q: \max \left(\mathbb{M}_{2 n}\left(\mathbb{M}_{2 n}\right)\right) \rightarrow \max \left(F_{n}\right)\left\|_{\mathrm{cb}}=\right\| Q \|=\frac{1}{2}(1+\sqrt{n})$. 
Taking the dual of this identity map, we obtain the third inequality.

Since an $\ell_{\infty}$-sum of Q-spaces is also a Q-space, we obtain the next corollary.

Corollary 2.2. There is a $Q$-space which is not exact.

Problem 2.3. What is the asymptotic behaviour of the constant

$$
\sup \{\operatorname{ex}(E): E \text { an } n \text {-dimensional Q-space }\}
$$

as $n$ tends to infinity?

\section{A Q-space which is not locally reflexive}

Definition 3.1. Let $C \geqslant 1$ be a constant. We say an operator space $X$ is $C$-locally reflexive if for any finite-dimensional subspaces $E \subset X^{* *}$ and $F \subset X^{*}$ and any $\varepsilon>0$, there is a map $\varphi: E \rightarrow X$ with $\|\varphi\|_{\mathrm{cb}}\langle C+\varepsilon$ such that $\langle\varphi(e), f\rangle=\langle e, f\rangle$ for all $e \in E$ and $f \in F$. We say an operator space $X$ is locally reflexive if $X$ is $C$-locally reflexive for some constant $C$.

We note that a $C^{*}$-algebra is 1-locally reflexive if it is locally reflexive and that a subspace of a $C$-locally reflexive operator space is also $C$-locally reflexive. See [5] for details. Now, let us construct a Q-space which is not locally reflexive. First, we need a lemma due to Oikhberg. Let us recall that the cb version of the Banach-Mazur distance between two completely isomorphic operator spaces $X$ and $Y$ is defined by

$d_{\mathrm{cb}}(X, Y)=\inf \left\{\|\varphi\|_{\mathrm{cb}}\left\|\varphi^{-1}\right\|_{\mathrm{cb}}: \varphi\right.$ a completely bounded isomorphism from $X$ onto $\left.Y\right\}$.

Put $d_{\mathrm{cb}}(X, Y)=\infty$ if $X$ and $Y$ are not completely isomorphic.

Lemma 3.2 (Lemma 3.4 from [15]). For every $C^{\prime}>0$, there is a finite-dimensional subspace $F \subset \max (\mathbb{B}(H))$ such that $d_{\mathrm{cb}}(F, G)>C^{\prime}$ for all $n$ and $G \subset \max \left(\mathbb{M}_{n}\right)$.

Taking the dual of the inclusion $F \subset \max (\mathbb{B}(H))$ we obtain a complete metric surjection $q: \min \left(S_{1}\right) \rightarrow E$, where $E=F^{*}$. By the above lemma and a small perturbation argument, we obtain the following lemma.

Lemma 3.3. For every $C^{\prime}>0$ there is a finite-rank complete metric surjection $q: \min \left(S_{1}\right) \rightarrow E$ such that $d_{\mathrm{cb}}(E, F /(\operatorname{ker} q \cap F))>C^{\prime}$ for all finite-dimensional subspaces $F \subset \min \left(S_{1}\right)$.

We now prove the following lemma.

Lemma 3.4. For every $C>0$ there is a $Q$-space which is not $C$-locally reflexive.

Proof. Fix $C^{\prime}>C$ and take a finite-rank complete metric surjection $q: \min \left(S_{1}\right) \rightarrow E$ as in Lemma 3.3. Let $\left\{F_{n}\right\}$ be an increasing sequence of finite-dimensional subspaces of $\min \left(S_{1}\right)$ such that $\overline{\cup F_{n}}=\min \left(S_{1}\right)$ and $q\left(F_{1}\right)=E$. Let $E_{n}=F_{n} /\left(\operatorname{ker} q \cap F_{n}\right)$ and let $\varphi_{n}: E_{n} \rightarrow E$ be the complete contraction induced by $\left.q\right|_{F_{n}}: F_{n} \rightarrow E$. By Lemma 3.3, we 
have $\left\|\varphi_{n}^{-1}\right\|_{\mathrm{cb}}>C^{\prime}$ for all $n$. On the other hand, it can be seen that $\lim _{n \rightarrow \infty}\left\|\varphi_{n}^{-1}\right\|_{k}=1$ for all $k \in \mathbb{N}$. Finally, let $X$ be an operator space defined by

$$
X=\left\{\left(x_{n}\right) \in\left(\prod E_{n}\right)_{\ell_{\infty}}: \lim _{n \rightarrow \infty} \varphi_{n}\left(x_{n}\right) \text { exists in } E\right\} .
$$

Since all the $E_{n}$ are Q-spaces, $X$ is also a Q-space. We will show that $X$ is not $C$-locally reflexive. There is a natural map $\varphi: X \rightarrow E$ defined by $\varphi\left(\left(x_{n}\right)\right)=\lim \varphi_{n}\left(x_{n}\right)$. For each $n$, define $\psi_{n}: E \rightarrow X$ by

$$
\psi_{n}(x)=\left(0, \ldots, 0, \varphi_{n}^{-1}(x), \varphi_{n+1}^{-1}(x), \ldots\right) .
$$

Since $\varphi_{m}^{-1} \circ \varphi_{n}: E_{n} \rightarrow E_{m}$ is completely contractive for all $m \geqslant n$, we have

$$
\lim _{n \rightarrow \infty}\left\|\psi_{n}\right\|_{k}=\lim _{n \rightarrow \infty}\left\|\varphi_{n}^{-1}\right\|_{k}=1
$$

for all $k \in \mathbb{N}$. Let $\psi: E \rightarrow X^{* *}$ be a cluster point of the sequence $\left\{\psi_{n}\right\}_{n}$ in the pointweak* topology. Then, by the previous argument, we have $\|\psi\|_{\mathrm{cb}} \leqslant 1$. Since $\varphi \circ \psi_{n}=\operatorname{id}_{E}$ for all $n$, we have $\varphi^{* *} \circ \psi=\operatorname{id}_{E}$. Now, suppose that $X$ is $C$-locally reflexive. Since $\varphi: X \rightarrow E$ is of finite rank, applying the local reflexivity to the complete contraction $\psi: E \rightarrow X^{* *}$, we obtain a map $\theta: E \rightarrow X$ with $\|\theta\|_{\mathrm{cb}}<C^{\prime}$ such that $\varphi \circ \theta=\mathrm{id}_{E}$. Let $\theta_{n}: E \rightarrow E_{n}$ be the ' $n$th coordinate' of $\theta$. Then, by the definition of $\varphi$, we have

$$
\lim _{n \rightarrow \infty} \varphi_{n} \circ \theta_{n}(x)=x
$$

for all $x \in E$. Since $E$ is finite dimensional, we have

$$
\limsup _{n \rightarrow \infty}\left\|\varphi_{n}^{-1}\right\|_{\mathrm{cb}} \leqslant \limsup _{n \rightarrow \infty}\left(\left\|\theta_{n}\right\|_{\mathrm{cb}}+\operatorname{dim}(E)\left\|\varphi_{n}^{-1}-\theta_{n}\right\|\right) \leqslant\|\theta\|_{\mathrm{cb}}<C^{\prime} .
$$

This contradicts the choice of $E$.

Theorem 3.5. There is a $Q$-space which is not locally reflexive.

Proof. For each $n$, there is a Q-space $X_{n}$ which is not $n$-locally reflexive by Lemma 3.4. Define a Q-space $Y$ by $Y=\left(\bigoplus X_{n}\right)_{c_{0}}$. It is easy to see that $Y$ is not locally reflexive.

Problem 3.6. In the proof of Lemma 3.4, it can be seen that $\operatorname{ex}(X)=\sup \operatorname{ex}\left(E_{n}\right)$. Can we control this value?

\section{Crude representability and local reflexivity}

Let $\left(\bigoplus X_{n}\right)_{\ell_{1}}$ be the $\ell_{1}$-direct sum of a sequence $\left\{X_{n}\right\}$ of operator spaces. We equip $\left(\bigoplus X_{n}\right)_{\ell_{1}}$ with the natural operator space structure (see pp. 34-36 in [18]). $\left(\bigoplus X_{n}\right)_{\ell_{1}}$ is then an operator space with the following properties. If $\varphi_{n}: X_{n} \rightarrow \mathbb{B}(H)$ is a complete contraction for all $n$, then $\varphi:\left(\bigoplus X_{n}\right)_{\ell_{1}} \ni\left(x_{n}\right) \mapsto \sum \varphi_{n}\left(x_{n}\right) \in \mathbb{B}(H)$ is a complete contraction. We have a completely isometric identity $\left(\bigoplus X_{n}\right)_{\ell_{1}}^{*}=\left(\prod X_{n}^{*}\right)_{\ell_{\infty}}$; and if $Y_{n} \subset X_{n}$ for all $n$, then we have $\left(\bigoplus Y_{n}\right)_{\ell_{1}} \subset\left(\bigoplus X_{n}\right)_{\ell_{1}}$ completely isometrically. When $X_{n}=X$ for all $n$, we simply denote $\left(\bigoplus X_{n}\right)_{\ell_{1}}$ by $\ell_{1}(X)$. 
Lemma 4.1. Let $X$ be a separable operator space and let $\left\{E_{n}\right\}$ be an increasing sequence of finite-dimensional subspaces of $X$ such that $\overline{U E_{n}}=X$. If $Y=\left(\bigoplus E_{n}\right)_{\ell_{1}}$ is $C$-locally reflexive, then so is $X$.

Proof. We follow the construction due to Johnson [9]. Let $q: Y \rightarrow X$ be a complete metric surjection defined by $q\left(\left(x_{n}\right)\right)=\sum_{n=1}^{\infty} x_{n}$. Fix a free ultrafilter $\mathcal{U}$ on $\mathbb{N}$ and define a map $r: Y^{*} \rightarrow X^{*}$ by

$$
\langle r(f), x\rangle=\lim _{\mathcal{U}}\left\langle f_{n}, x\right\rangle
$$

for all $f=\left(f_{n}\right) \in Y^{*}=\left(\prod E_{n}^{*}\right)_{\ell_{\infty}}$ and $x \in \bigcup E_{n}$. Then, $r$ is a well-defined complete contraction and $r \circ q^{*}=\mathrm{id}_{X^{*}}$. To prove that $X$ is $C$-locally reflexive, we give ourselves finite-dimensional subspaces $E \subset X^{* *}$ and $F \subset X^{*}$ and $\varepsilon>0$. Let $\tilde{E}=r^{*}(E) \subset Y^{* *}$ and let $\tilde{F}=q^{*}(F) \subset Y^{*}$. Since $Y$ is $C$-locally reflexive, there is a map $\varphi: \tilde{E} \rightarrow Y$ with $\|\varphi\|_{\mathrm{cb}} \leqslant C+\varepsilon$ such that $\langle\varphi(\tilde{e}), \tilde{f}\rangle=\langle\tilde{e}, \tilde{f}\rangle$ for all $\tilde{e} \in \tilde{E}$ and $\tilde{f} \in \tilde{F}$. Now, define $\psi: E \rightarrow X$ by $\psi=q \circ \varphi \circ\left(\left.r^{*}\right|_{E}\right)$. Then, we have $\|\psi\|_{\mathrm{cb}} \leqslant C+\varepsilon$ and

$$
\begin{aligned}
\langle\psi(e), f\rangle & =\left\langle\varphi\left(r^{*}(e)\right), q^{*}(f)\right\rangle \\
& =\left\langle r^{*}(e), q^{*}(f)\right\rangle \\
& =\left\langle e, r \circ q^{*}(f)\right\rangle \\
& =\langle e, f\rangle
\end{aligned}
$$

for all $e \in E$ and $f \in F$. This completes the proof.

Lemma 4.2 (Theorem 4.3 in [7]). An operator space $X$ is locally reflexive if every separable subspace of $X$ is locally reflexive.

Proof. The 'isometric' version of this lemma has been proved in [7]. Observe that the assumption implies that there is a constant $C$ so that every separable subspace of $X$ is $C$-locally reflexive. Now the proof of $C$-local reflexivity of $X$ is almost same as the proof of Theorem 4.3 in [7].

Let $Z$ and $X$ be operator spaces. We say $X$ is crudely representable in $Z$ if there is a constant $C$ such that for any finite-dimensional subspace $E$ of $X$, there is a subspace $F$ of $Z$ with $d_{\mathrm{cb}}(F, E)<C$.

Theorem 4.3. Let $Z$ be an operator space such that $Z$ contains a completely isomorphic copy of $\ell_{1}(Z)$. Assume that $Z$ is locally reflexive. If $X$ is an operator space which is crudely representable in $Z$, then $X$ is locally reflexive.

Proof. By Lemma 4.2, we may assume that $X$ is separable. Take an increasing sequence $\left\{E_{n}\right\}$ of finite-dimensional subspaces of $X$ with $\overline{\bigcup E_{n}}=X$. Since $X$ is crudely representable in $Z, Y=\left(\bigoplus E_{n}\right)_{\ell_{1}}$ can be embedded into $\ell_{1}(Z)$ completely isomorphically. Since a subspace of locally reflexive operator space is also locally reflexive, by Lemma 4.1, we are done. 
Remark 4.4. In [10], Junge has proved that the operator space $S_{1}$ of trace class operators satisfies the assumption of Theorem 4.3 and the consequence is already known [6]. There is a locally reflexive operator space $X$ such that $\ell_{1}(X)$ is not locally reflexive. Indeed, if $V$ is a separable operator space which is not locally reflexive and $\left\{E_{n}\right\}$ is an increasing sequence of finite-dimensional subspaces with $\overline{U E_{n}}=V$, then $X=\left(\bigoplus E_{n}\right)_{c_{0}}$ is locally reflexive, but $\ell_{1}(X)$ is not locally reflexive. This answers a question raised by Le Merdy (personal communication). There is an ' $\ell_{\infty}$-version' of Theorem 4.3 (use Lusky's construction [14] at Lemma 4.1), but it seems that for only few operator spaces $X, \ell_{\infty}(X)$ is locally reflexive.

Problem 4.5. Are exact operator spaces necessarily locally reflexive?

It has been shown in Corollary 4.8 in [7] that every 1-exact operator space is 1-locally reflexive.

Acknowledgements. The author is supported by JSPS. The author thanks Professor Gilles Pisier for introducing him to the problem and for a stimulating conversation.

\section{References}

1. D. Blecher, The standard dual of an operator space, Pacific J. Math. 153 (1992), 15-30.

2. D. Blecher And V. Paulsen, Tensor products of operator spaces, J. Funct. Analysis 99 (1991), 262-292.

3. J. De Cannière and U. HaAgerup, Multipliers of the Fourier algebras of some Lie groups and their discrete subgroups, Am. J. Math. 107 (1985), 455-500.

4. E. Effros And Z.-J. Ruan, A new approach to operator spaces, Can. Math. Bull. 34 (1991), 329-337.

5. E. Effros And Z.-J. RuAn, Mapping spaces and liftings for operator spaces, Proc. Lond. Math. Soc. 69 (1994), 171-197.

6. E. Effros, M. Junge And Z.-J. RuAn, Integral mappings and the principle of local reflexivity for non-commutative $L^{1}$-spaces, Ann. Math. (2) 151 (2000), 59-92.

7. E. Effros, N. OzaWA AND Z.-J. RUAN, On injectivity and nuclearity for operator spaces, Duke Math. J., in press.

8. U. HaAgerup and G. Pisier, Bounded linear operators between $C^{*}$-algebras, Duke Math. J. 71 (1993), 889-925.

9. W. B. Johnson, A complementary universal conjugate Banach space and its relation to the approximation problem, Israel J. Math. 13 (1972), 301-310.

10. M. JUnGE, Factorization theory for spaces of operators (Habilitationsschrift, Universität Kiel, 1996).

11. E. KirChBerG, On non-semisplit extensions, tensor products and exactness of group $C^{*}$-algebras, Invent. Math. 112 (1993), 449-489.

12. E. KirchBerg, On subalgebras of the CAR-algebra, J. Funct. Analysis 129 (1995), 3563.

13. C. Lance, On nuclear $C^{*}$-algebras, J. Funct. Analysis 12 (1973), 157-176.

14. W. Lusky, A note on Banach spaces containing $c_{0}$ or $C_{\infty}$, J. Funct. Analysis 62 (1985), $1-7$.

15. T. OikHBerg, Subspaces of maximal operator spaces, preprint.

16. G. PIsier, Remarks on complemented subspaces of von Neumann algebras, Proc. R. Soc. Edinb. A 121 (1992), 1-4. 
17. G. Pisier, Exact operator spaces, in Recent Advances in Operator Algebras, Orléans 1992, Astérisque Soc. Math. France 232 (1995), 159-186.

18. G. PISIER, Non-commutative vector valued $L_{p}$-spaces and completely $p$-summing maps, Astérisque Soc. Math. France 247 (1998), vi + 131 pp.

19. A. G. Robertson, A non-extendible positive map on the reduced $C^{*}$-algebra of a free group, Bull. Lond. Math. Soc. 18 (1986), 389-391.

20. A. G. Robertson And R. Smith, Lifting and extension of maps on $C^{*}$-algebras, $J$. Operat. Theory 21 (1989), 117-131.

21. R. Sмith, Completely bounded maps between $C^{*}$-algebras, J. Lond. Math. Soc. 27 (1983), 157-166. 